\author{
Buribayeva M.S., Kunakbayeva A., Abebova L., *Irmukhametova G.S., \\ Muldagulova K.B., Mun G.A. \\ Al-Farabi Kazakh National University, Kazakhstan, Almaty \\ *E-mail: i_galiya@mail.ru
}

\title{
Transdermal study of hydrogel ointments and bandages with antituberculosis activity
}

\begin{abstract}
Transdermal study for hydrogel ointments and bandages based on polyvinylpirrolidone was conducted for application as transdermal therapeutic drug delivery systems for skin tuberculosis treatment. The ointments and bandages based on polyvinylpyrrolidone (PVP) and carbomer with drugs isoniazid and ethambutol were obtained. The transdermal characteristics were investigated using cellulose membrane and pig skin. The experiment was carried out by the device Franz cell and diffusion coefficient (D), permeability (P) and permeation rates (F) of the drugs were calculated. It was established that the rate of isoniazid penetration through the skin faster than that for ethambutol which is related to the structure peculiarities of drugs. The higher transdermal activity of hydrogel ointments was shown in comparison with hydrogel dressings. Also the prolongation effect of developed transdermal drug delivery systems based on PVP and carbomer was observed.
\end{abstract}

Key words: transdermal therapeutic system, drug delivery, polyvinylpyrrolidone, isoniazid, ethambutol, carbomer

\section{Introduction}

In recent years there has been an extreme rise in tuberculosis worldwide. In modern medicine as one of the basic issues is the creation of new forms of drugs with high therapeutic activity, for controlled release of drugs and their exact delivery to the site of the pathological process. Due to this, drugs (DS) based polymers are promising, because they provide prolonged action. Such important drugs include DS used against tuberculosis, because treatment of tuberculosis takes a very long time. The reason is excessively accumulation of DS that's why minor allergic reactions are manifested. In connection with this the creation of hydrogel ointments based on biopolymers as transdermal systems are important in medicine application. Almost all medications which are used to treat tuberculosis can cause toxic side effects. Therefore, there is an obvious need for new biopolymers such as hydrogel based on polyvinylpyrrolidone (PVP). At the same time, by research of their transdermal systems through the skin, their use in medicine could be predicted [1].
Skin of an average adult body covers a surface of approximately $2 \mathrm{~m}^{2}$ and receives about one-third of the blood circulating through the body. Skin contains an uppermost layer, epidermis which has morphologically distinct regions; basal layer, spiny layer, stratum granulosum and upper most stratum corneum, it consists of highly cornified (dead) cells embedded in a continuous matrix of lipid membranous sheets. These extracellular membranes are unique in their compositions and are composed of ceramides, cholesterol and free fatty acids.

The human skin surface is known to contain, on an average, 10-70 hair follicles and 200-250 sweat ducts on every square centimeters of the skin area. It is one of the most readily accessible organs of the human body. The potential of using the intact skin as the port of drug administration to the human body has been recognized for several decades, but skin is a very difficult barrier to the ingress of materials allowing only small quantities of a drug to penetrate over a period of time. Transdermal drug delivery - the delivery of drugs across the skin and into systemic circulation - is distinct from topical drug penetra-

Printed in Kazakhstan 
tion, which targets local areas. Transdermal drug delivery takes advantage of the relative accessibility of the skin. [3]

The drug has to go through these structural layers, encountering several lipophilic and hydrophilic barriers in the dermis, where absorption into the systemic circulation is rapid because of the large capillary bed. Dermis is the thickest of the skin layer (5.3 $\mathrm{mm}$ ) and include hair follicles, sweat glands, nerve endings and blood and lymph vessels. The dermis acts as a systemic absorption of drugs.

The more common pathway through the skin is via the intercellular route. Drugs crossing the skin by this route must pass through the small spaces be- tween the cells of the skin, making the route more tortuous. Although the thickness of the stratum corneum is only about $20 \mu \mathrm{m}$, the actual diffusional path of most molecules crossing the skin is on the order of $400 \mu \mathrm{m}$. The 20 -fold increase in the actual path of permeating molecules greatly reduces the rate of drug penetration (Fig. 2).

A less important pathway of drug penetration is the follicular route. Hair follicles penetrate through the stratum cornea, allowing more direct access to the dermal microcirculation. However, hair follicles occupy only $1 / 1,000$ of the entire skin surface area. Consequently, very little drug actually crosses the skin via the follicular route. [6]

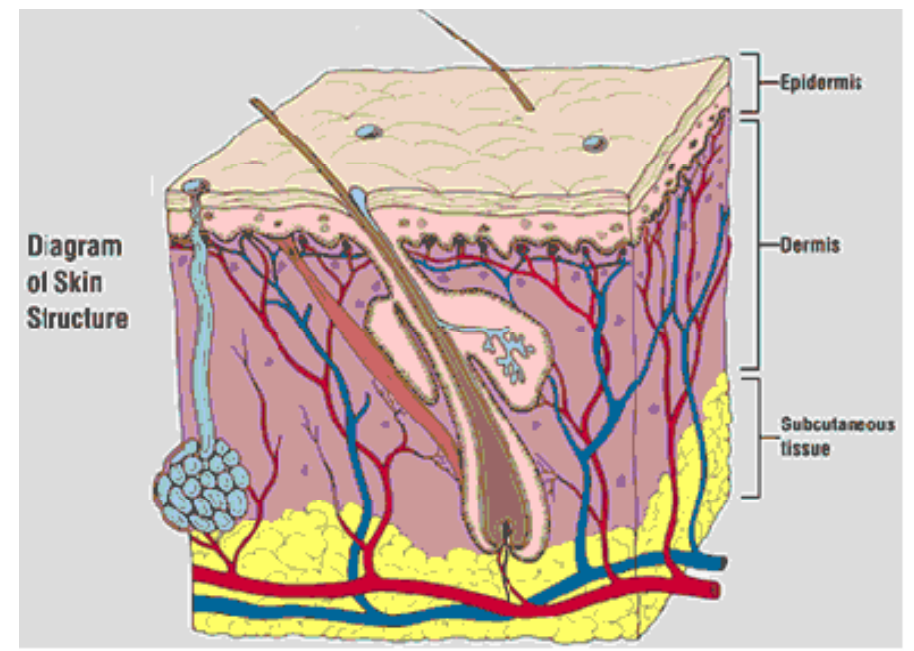

Figure 1 - Diagram of skin structure [4]

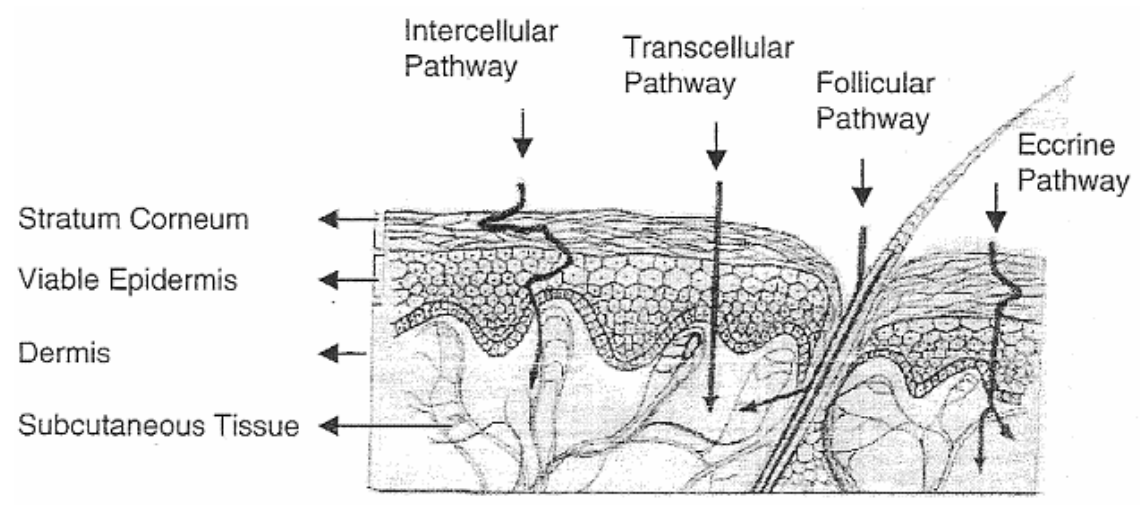

Figure 2 - Various routes of drug absorption [5] 


\section{Materials and methods}

Polyvinylpirrolidone $\left(\mathrm{M}_{\mathrm{n}}=1000000\right)$, polyethylene glycole $\left(M_{n}=2000\right)$ were purchased from Merck Chemie and used as received, agar-agar micribiologycal and gellan were purchased from Sigma Aldrich and was used without purification. Carbomer was purchased from Aromamaslo and was used as received.

Hydrogel ointment preparation. The carbopol was placed into distilled water for swelling during the 2-3 minutes. Then the mixture was thoroughly stirred on magnetic stirrer then gradually added to the aqueouse solutions of drugs isoniazid $(0,06 \mathrm{~g} /$ $\mathrm{ml})$ and ethambutol $(0,1 \mathrm{~g} / \mathrm{ml})$ and stirred until getting homogeneous consistency. Then, the solution of $50 \% \mathrm{NaOH}$ was dropwise added to the mixture in order to increase the $\mathrm{pH}$ from 3 to 7 and thicken the hydrogel ointment.

Hydrogel dressings preparation. PVP was completely dissolved in distilled water at $40-50^{\circ} \mathrm{C}$ during the 30-40 minutes. Agar-agar was dissolved in distilled water at $70-80^{\circ} \mathrm{C}$. In case of using gellan as a thickener no temperature was used for gellan dissolving process. Then, the dissolved agar-agar (or gellan) was mixed with PVP and PEG was added under discontinuous mixing. After, the solution of drugs isoniazid $(0,06 \mathrm{~g} / \mathrm{ml})$ and ethambutol $(0,1 \mathrm{~g} / \mathrm{ml})$ were added to prepared mixtures and stirred at $40-50^{\circ} \mathrm{C}$ until getting to homogeneous mixture. Prepared mixtures were poured onto the plastic substrate, cooled to room temperature, sealed and sent to radiation crosslinking and sterilization. The composition of the hydrogel dressing is presented in Table 8 .

Transdermal study. The penetration ability of drugs was studied using cellulose membrane and pig ears skin. Dialysis tubing MWCO 12-14 kDa (Medicell Int. Ltd, UK) was used for this purpose. Pig ears thickness about 500-600 microns were purchased from Almaty (individuals age 3-4 months; weight $25-30 \mathrm{~kg}$ ). Pig ears were stored at $20{ }^{\circ} \mathrm{C}$ until use. Before each experiment, they were warmed to room temperature. The full-thickness of pig ear skin samples was measured before diffusion assay. The mean thickness of intact and excoriated skin samples was respectively $1.07 \pm 0.10 \mathrm{~mm}$ and $0.92 \pm 0.05$.

The Franz diffusion cells system used for determined penetration of drug. Franz cell receptor compartment was filled with $35 \mathrm{ml}$ of $0.2 \mathrm{M}$ phosphate pH 6,86 buffer solution (Sigma-Aldrich) homogenized by magnetic stirring $(400 \mathrm{rpm})$ and maintained at $37^{\circ} \mathrm{C}$. Skin biopsies were placed between the donor and the receptor compartment ensuring that the dermal face was in contact with the receptor medium, whereas the epidermal face was turned to the donor compartment. The whole device was then fixed with a clamp.

Diffusion of drugs into the receptor compartment of Franz cell was measured spectroscopically by UV spectrometer Philips 8715 Model PU, at a wavelength of isoniazid $\chi=270 \mathrm{~nm}$ and ethambutol $\chi=275 \mathrm{~nm}$, spectra were recorded in the wavelength range $190-500 \mathrm{~nm}$.

\section{Results}

The modern pharmaceutical technology as one of the basic tasks considers the establishment of new hydrogel ointments and dressings with drugs based on biocompatible polymers such as polyvinyl pyrrolidone, polyvinyl alcohol. These drug delivery systems can have high therapeutic activity and used for controlled drug release and its specific delivery directly to the pathological process. This is important as there are groups of serious diseases, which could be overcome, not because of drug effect itself, but also depend on the method of drug delivery into the body. In this research the transdermal properties of hydrogel ointments and dressings with DS through porcine skin was studied.

In this paper the hydrogel ointment and bandages based in biocompatible polymers for application as a transdermal therapeutic delivery systems with antituberculosis drugs isoniazid and ethambutol were developed and its transdermal activity was studied. As we can see from the figures 3 and 4, the amount of DS permeated through the skin in both cases increases by increasing of its concentration in the ointment. In this case, the permeability of isoniazid through the skin is much better than ethambutol, which is related to the chemical structure of drugs. It should be noted that isoniazid has less branched structure, and it belongs to the most effective drugs of the first class. Ointment with isoniazid DS are characterized by significant physiological indifference, the ability to be absorbed by intact skin and a relatively easiness of release of the incorporated drug substances. The reason is that they are more hydrophobic than ethambutol.

Drug dialysis through a cellulose membrane was investigated and based on obtained results the diffusion coefficients (D), the degree of penetration of drug $(\mathrm{P})$ and the flow rate of drug $(\mathrm{F})$ were calculated. These calculations are presented in Table 1. Calculated results from table confirm the results shown in Figures 3-4. 


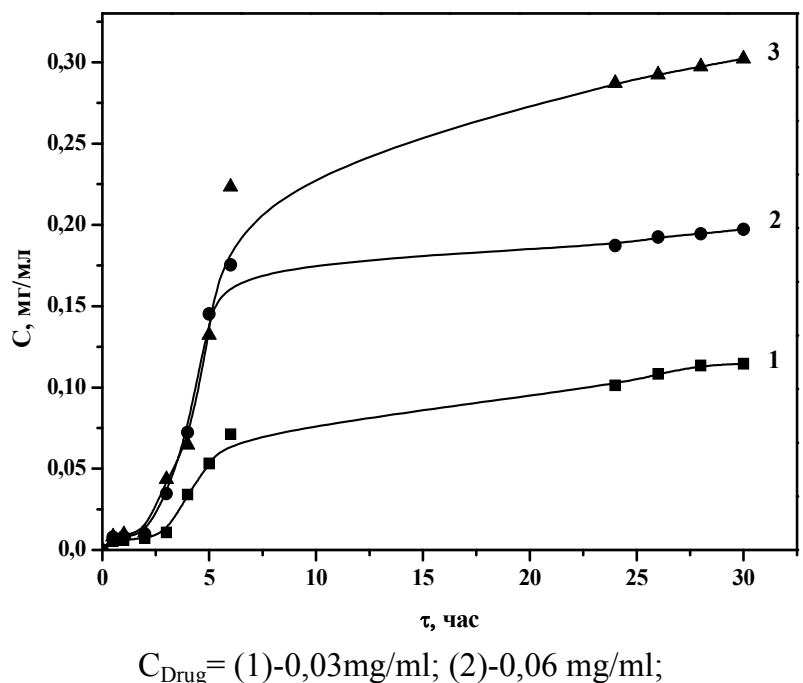

(3) $-0,09 \mathrm{mg} / \mathrm{ml}$

Figure 3 - Kinetics of isoniazid penetration from ointment through the pig skin

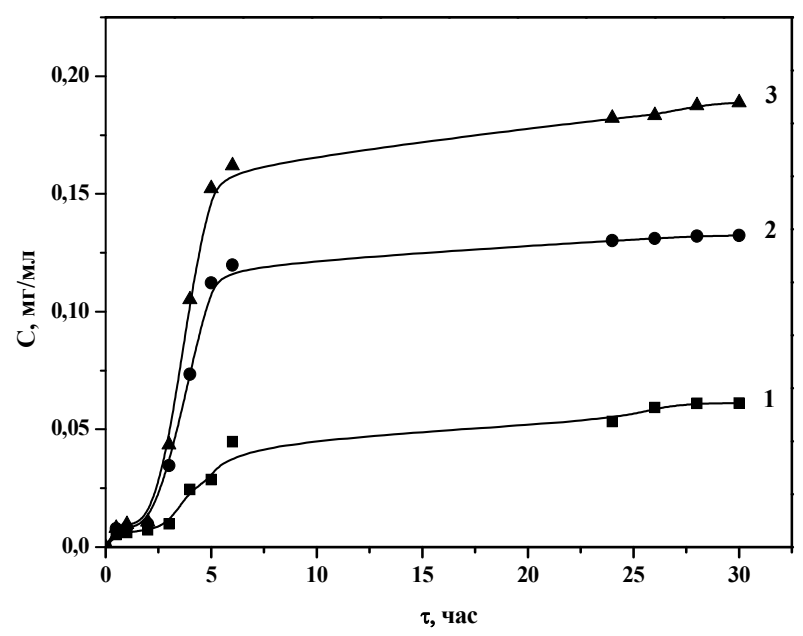

$\mathrm{C}_{\text {Drug }}=(1)-0,05 \mathrm{mg} / \mathrm{ml} ;(2)-0,1 \mathrm{mg} / \mathrm{ml}$; (3) $-0,15 \mathrm{mg} / \mathrm{ml}$

Figure 4 - Kinetics of ethambutol penetration ointment through the pig skin

Table 1 - Penetration parameters of drugs from cellulose membrane and skin

\begin{tabular}{|l|c|c|c|c|c|c|}
\hline \multirow{2}{*}{ Drug } & \multirow{2}{*}{$\begin{array}{c}\text { Concentration, } \\
\mathrm{g} / \mathrm{ml}\end{array}$} & \multicolumn{2}{|c|}{ Through membrane } & \multicolumn{3}{|c|}{ Through pig skin } \\
\cline { 2 - 7 } & $\begin{array}{c}\mathrm{P}, \\
\mathrm{sm}^{2} \times \mathrm{sec}^{-1}\end{array}$ & $\begin{array}{c}\mathrm{F}, \\
\mathrm{mg} \times \mathrm{min}\end{array}$ & $\begin{array}{c}\mathrm{D}, \\
\mathrm{sec}^{1} \times \mathrm{cm}^{2} \times \mathrm{mm}\end{array}$ & $\begin{array}{c}\mathrm{F}, \\
\mathrm{mg} \times \mathrm{min}\end{array}$ & $\begin{array}{c}\mathrm{P}, \\
\mathrm{sm}^{2} \times \mathrm{sec}^{-1}\end{array}$ \\
\hline \multirow{3}{*}{ isoniazid } & 0,03 & $12.52 \times 10^{-2}$ & $2,19 \times 10^{-2}$ & 3.6958 & $1.94 \times 10^{-2}$ & $4.52 \times 10^{-2}$ \\
\cline { 2 - 7 } & 0,06 & $15.28 \times 10^{-2}$ & $2,98 \times 10^{-3}$ & 5.0567 & $4.34 \times 10^{-4}$ & $9.81 \times 10^{-2}$ \\
\cline { 2 - 7 } & 0,09 & $20.52 \times 10^{-2}$ & $3.77 \times 10^{-3}$ & 8.9861 & $5.03 \times 10^{-3}$ & $16.04 \times 10^{-3}$ \\
\hline \multirow{3}{*}{ ethambutol } & 0,05 & $14.09 \times 10^{-2}$ & $3,84 \times 10^{-3}$ & 1.1646 & $2.79 \times 10^{-2}$ & $6.31 \times 10^{-1}$ \\
\cline { 2 - 7 } & 0,1 & $14.72 \times 10^{-2}$ & $3,94 \times 10^{-3}$ & 1.1838 & $5.33 \times 10^{-2}$ & $9.83 \times 10^{-1}$ \\
\cline { 2 - 7 } & 0,15 & $22.56 \times 10^{-2}$ & $5,62 \times 10^{-3}$ & 3.6487 & $8.44 \times 10^{-2}$ & $10.18 \times 10^{-1}$ \\
\hline
\end{tabular}

For investigation the transdermal activity of hydrogel dressings the bandages (adhesive area of 2 $\mathrm{cm}^{2}$ ) based on PVP and PVA with a therapeutic concentration of DS isoniazid and ethambutol (therapeutic doses of isoniazid $1,2 \mathrm{~g} /$ day; ethambutol $2 \mathrm{~g}$ /day) were prepared.

Investigation of DS concentration impact on transdermal activity of hydrogel bandages is shown in figures 5-6. It is seen that the amount of drug isoniazid and ethambutol, permeated through the skin is equal to zero in the interval of 0-30 minutes from the start of the experiment. This segment of the curve is called the «lag time», its describes the time needed for DS penetrate through the layers of skin. It can be seen that with increasing DS concentration in the hydrogel bandages the number of penetrated DS increases. The maximum amount of penetrated DS from PVP agar-agar based dressings is $0.165 \mathrm{mg} / \mathrm{mL}$ for isoniazid and $0.12 \mathrm{mg} / \mathrm{mL}$ for ethambutol during 23-24 hours. And from PVP-gellan based dressings $0.13 \mathrm{mg} / \mathrm{mL}$ for isoniazid and $0.08 \mathrm{mg} / \mathrm{mL}$ for ethambutol during 23-24 hours.

It can be seen from obtained results, the penetration ability of isoniazid through the skin much better that that is for ethambutol. It can be related to chemicak structure of drugs. The isoniazid has branched structure and it's more effective first class DS.

Comparison study of transdermal activity of hydrogel ointment and dressings showed that the drug release rate from ointment higher than that is for hydrogel dressing (figure 7) but prolongation effect of dressings is better. Also it can be seen from the picture 7 that the transdermal activity of PVP and agaragar based dressing is higher the PVP and gellan based formulation. It can be related to higher swelling degree of PVP and agar-agar hydrogel dressing. 


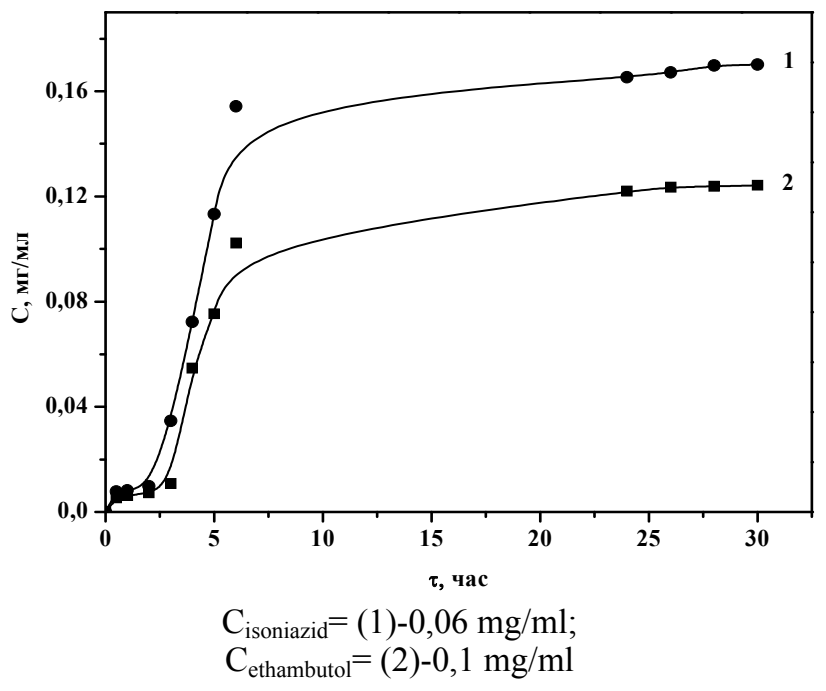

Figure 5 - Kinetics of drugs penetration from PVP and agar-agar based hydrogel dressing through the pig skin

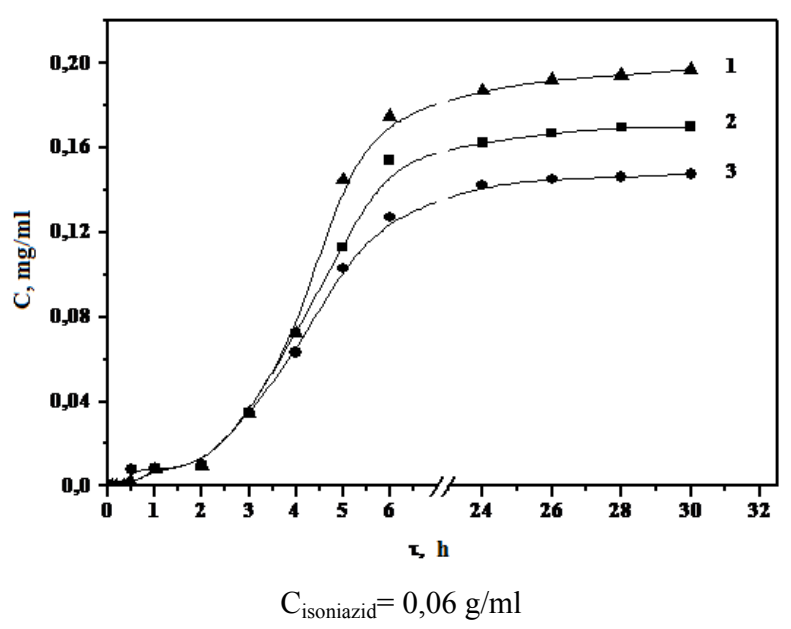

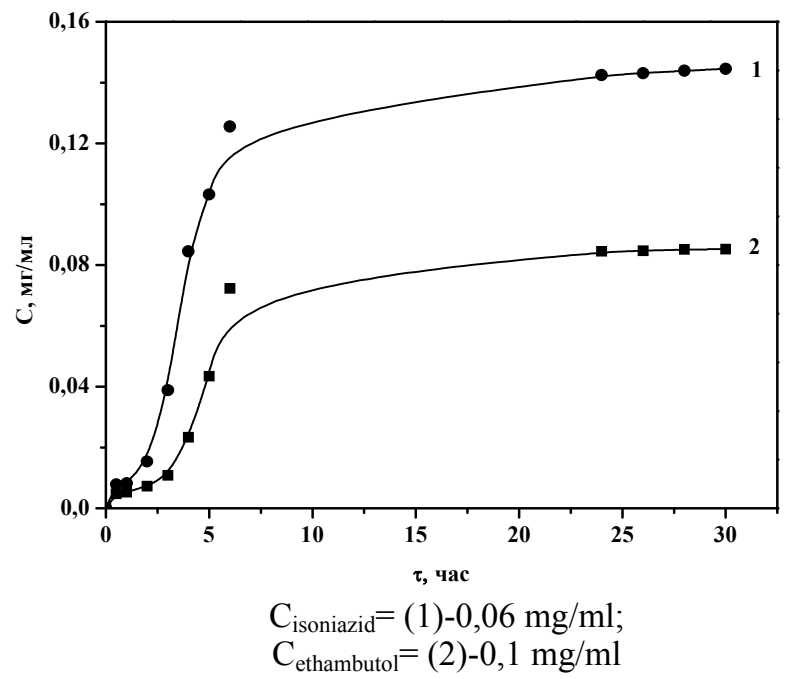

Figure 6 - Kinetics of drugs penetration from PVP and gellan based hydrogel dressing through the pig skin

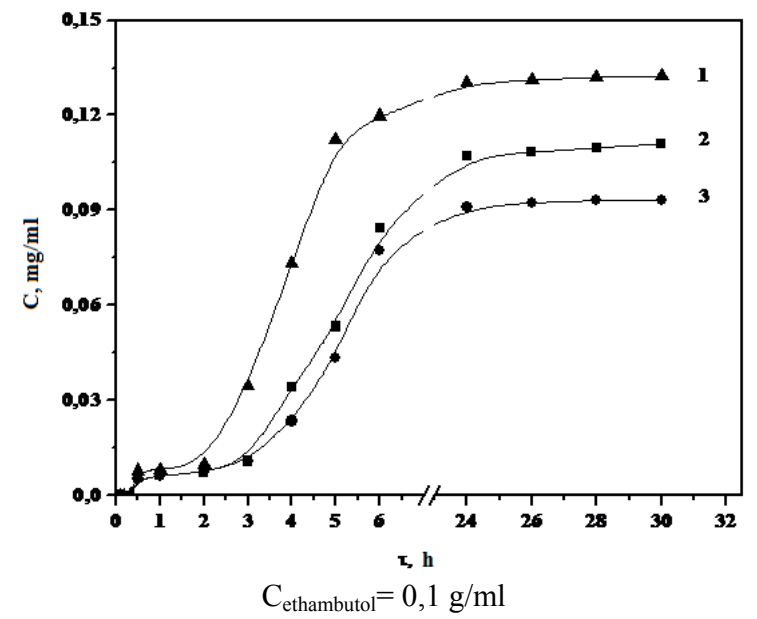

1 - hydrogel ointment, 2 - hydrogel dressing based on PVP and agar-agar, 3 - hydrogel dressing based on PVP and gellan

Figure 7 - Penetration kinetics of DS from hydrogel dressings and ointment.

\section{Conclusion}

Thus in present study the transdermal activity of hydrogel ointments and bandages based on PVP with antituberculosis drugs isoniazid and ethambutol of different concentrations was investigated. It is shown that both drugs penetrate the skin at a rate sufficient to achieve the daily therapeutic dose. Moreover the rate of penetration of isoniazid higher than the rate of penetration of ethambutol, which is related to chemical structure of DS. Investigation of transdermal activity of two types of hydrogel dressings showed that PVP and agar-agar based formulations shows higher rate of drug penetration through the skin. The comparison of hydrogel ointments and dressings resulted in better prolongation effect of dressings but the smaller rate of DS penetration and as a result its lower transdermal activity.

\section{References}

1 Jasti BR, Abraham W, Ghosh TK. Transdermal and Topical drug delivery systems. In: Ghosh TK, Jasti BR, editors. Theory and Practice of Contemporary Pharmaceutics. 1st ed. Florida: CRC Press; 2005. p. 423-53. 
2 Baker H. The skin as barrier. In: Rook A, Wilkinson DS, Ebling FJG, Champion RH, Burton JL, eds. Text Book of Dermatology. Vol. I, 4th ed. Oxford: Blackwell Scientific Publications, 1986:35566.

3 Barr M. Percutaneous absorption, J Pharm Sci 1962; (61):395-409.

4 Chein YW. Advances in transdermal systemic drug delivery. Drugs of Future 1988; (13):343-62.
5 N. Blanchin, S. Desloires, L. Grappin, A.M. Guillermin, P. Lafon, A. Miele, Protocols in an occupational medical facility for the management of internal plutonium exposure incidents in a nuclear plant: development-application-analysis-validation from 1996 to 2002, Radioprotection 39 (2004) 59-75.

6 B.M. De Rey, H.E. Lanfranchi, R.L. Cabrini, Percutaneous absorption of uranium compounds, Environ. Res. 30 (1983) 480-491. 